\title{
Research on the Application of Skeleton Identification in the New Media Game
}

\author{
Jiajia Xu ${ }^{1}$, Jing Yan*², Haiyan Jiang ${ }^{3}$ \\ 1 Wuhan Institute of shipping Technology, Wuhan, 430050 \\ 2 State Grid Hubei Electric Power Company Limited Economic Research Institute, 430050, \\ 105908342@qq.com \\ 3 Wuhan University, Wuhan, 430072
}

Keywords: skeleton identification, interactive design, motion sensing game, Kinect motion sensing device

\begin{abstract}
With the advent of era of intelligence, human skeleton identification and capture have drawn more and more attention. Human skeleton identification is the tendency of game design in the new media. However, there is a lack of theory about how to integrate skeleton identification with game design, and about how to give full play to its technological advantages so as to improve the quality of game design in the new media. The paper, therefore, analyzes scientific and technological advantages in skeleton identification technology and its features, concludes and improves the application and scope of this technology in the game design in the new media, striving to enlighten and provide positive reference for the interactive game design based on skeleton identification.
\end{abstract}

\section{Introduction}

With the invention of the first computer in the world, communication barriers appear in the process. Thus, as the computer develops rapidly, human-computer interaction design tends to be humanized. As the increasing progress of computer technology, human-computer interaction has grown from the manual work initially to the natural human-computer interaction now. More and more users would like to abandon traditional tools like mouse and keyboard so as to enjoy the communication with computer in a more natural manner. It is fair that, direct interaction with computers enables users to feel more interested in this interaction. Based on this, the increasing advances in human-computer interaction has promoted the development of motion sensing game, which captures body movement of players by tracking the position of key nodes on the skeleton, and then, follows the action of players by the action analyzer. Unlike traditional games, motion sensing game is more suitable foe human's natural habits. So the capture technology of human skeleton data holds the key to motion sensing game.

\section{Development and rise of skeleton identification}

Thanks to the development of animation from the 1960s to 1970s, motion capture rises. And the concept of human motion capture was firstly proposed by The Walt Disney Company in America in order to improve the effect of animation. Later, people used light to project human body movements onto the screen, creating a real reference model for animation production. After the 1990s, the development of computer software as well as hardware put motion capture into place, and permeated various fields. With the increasingly mature of Internet technology, multi-media network has become a part of human life, while human-computer interaction has been shifted from the original manual work to advanced people-centered intelligent interaction. And motion sensing enables users to break the shackles of hardware operation. In the advance of natural human-computer interaction, a large amount of hardware comes out successively such as Helmet-Mounted 3D Displays, data glove, magic rings. In addition, more vivid user interface, wraparound sound effect, command language is more conducive to natural human-computer 
interaction.

At present, more and more researchers have devoted to the motion capture both at home and aboard. Alexandros detects body movements in the full range through many cameras, and then, makes vectorization for detected data, which relatively precisely identifies average body motions, while fails to catch motions timely because of too long time for acquiring a large number of data by many cameras. Hence, Balckbum et al. put forward another algorithm to preprocess sampling videos by dimensionality reduction, and then classified and identified motions according to similar motions in the template library in the way of pixel matching. Despite simple operation, the method has some limitations in the motion identification. After that, Jingen et al. used the human motion recognition of unconstrained video stream to match the similar features with high identification rate. And in the virtual games, developers domestically and internationally have realized the significance of skeleton identification for the future game development, therefore, human skeleton identification has attracted more and more attention. Present technologies lead players to enter the virtual environment of game by wearing cameras and other equipment in order to increase the sense of reality of players. Kinect, LeapMotion, Will and PsMove by Sony are popular motion sensing equipment, among them, Kinect is a more popular motion sensing input device, whose principle is to utilize infrared cameras to capture and track players' body movements in real time, so as to restore the game players' movement trails as much as possible in the motion sensing games.

\section{Skeleton identification}

Kinect is a motion sensing camera released by Microsoft in the E3 Exhibition in 2009. Unlike ordinary cameras, Kinect is more intelligent. The infrared light emitted by Kinect has two functions: The first is the 3D positioning of the overall room, and the second is to help cameras identify body movements with the help of infrared light. Moreover, with the upgrading of Kinect, the second generation of Kinect provides optimized skeleton tracking functions, which can track 6 intact skeletons and 25 joints of human body at most, improving the skeleton tracking furtherly. Kinect, identifies speech and determines positions with color camera, infrared transmitters, and infrared light, meanwhile, it uses Moving Touch、Kionix KXSD9 and triaxial accelerometer to ensure the precision of in-depth data collected by Kinect. Kinect adopts the Light Coding developed by PrimeScene Company, its most unique feature lies in the random and laser speckle without repetition, which can mark objects in the room, judges and identifies the positions of objects in the three-dimensional space in light of different speckle patterns. Kinect will capture the outline of the pattern, and then the specific details, it will acquire deep information and then build coordinates for human joints, finally, it will identify the changes of players' body movements in terms of changes in the coordinates of joints. And players in the most efficient regions near the Kinect will be given top priority. Any objects shaped like “大”( a Chinese character) will be marked as players by Kinect, that is the reasons for plays should open arms before some motion sensing games who will be scanned gradually from the head, hands, and other parts.

\section{The application of skeleton identification in the game and its troubles}

However, when E3 Exhibition is celebrated by many, there are many problems haunting people. First of all, unclear releasing time of these games makes players feel it is not a foreseeable time, some even doubt if they can witness these games in their lifetime. Second, there is a large gap between the final games and the promotion in the exhibition. All these problems make players feel disappointed. Why people have such troubles in the high-speed development of science and technology in 2015? There is no doubt about the bright prospect of motion sensing game, but now, it is not the mainstream, and the following is some problems.

First, average players cannot afford the costly motion sensing games. And it is easy to imagine that the prices of motion sensing games with multiplayers online will rise. The high prices greatly restrict the consumer groups of motion sensing games, directly affecting the profits for game companies. While the research and development of motion sensing games is based on a large 
number of professionals and vast funds. If the sales cannot be guaranteed, game companies will not have enough money to attract talents and make further research and development.

Second, technology is still an important factor limiting the development of motion sensing game. Despite a variety of motion sensing devices, there are also many problems, for instance, the playing environment is not realistic that players will feel a fit of dizziness, the motion sensing game cannot precisely identify the body movements. All these require developers to start from the real needs of players, and make further improvement in technology.

Eventually, the mainstream of the game market is the mobile game and game on the personal computers rather than motion sensing games. The reasons include that, the companies specialized in motion sensing games are relatively few, and almost all themes of motion sensing games are sports, virtually limiting the consumer groups.

Despite a lot of problems in motion sensing games, there is no doubt about its prospect. With the increasingly development of scientific and technological intelligence, more and more people will know and be fond of motion sensing games, which will greatly promote game developers to seize opportunities to develop hardware, and break through bottlenecks. At the same time, the development of science and technology will drive the price of hardware to drop, attracting more and more players. And as people demand more, the type of games will expand, and motion sensing game will permeate every fields like fitness, health, and teaching.

\section{Conclusions}

Skeleton identification replaces the traditional human-computer interaction with a new one by virtual reality via the Internet. And human-computer interaction is sure to be more user-friendly and natural. Human motion identification is definitely the only way to the friendly interaction and game is a deep interaction. Therefore, skeleton identification has a huge growth driver in the tremendous development of new media games, and has great potential to promote the new media games to reach a new high led by science and technology.

\section{References}

[1] Li Shiguo, Hua Meili, Jia Rui. New models in the product design-interactive design[J], packaging engineering, 2007(4).

[2] Jiang Xuezhi, Li Zhonghua. Research status of virtual reality at home and abroad. Journal of Liaoning Technical University, 2004,23(2).

[3] Gu Weihong, Min Kunlong, Zhang Xiaona. Study on the new data gloves and its gesture identification[J], Process Automation Instrumentation,2012,32(2).

[4] Zhao Qinping. Summarization of virtual reality[J], Science China (F edition: information science),2009(1).

[5] Liu Yating. Research on the application mode and development of virtual reality in new media[J], Today's Massmedia,2017(9). 